\title{
Rational Trigonometry in Higher Dimensions and a Diagonal Rule for 2-planes in Four-dimensional Space
}

\author{
Rational Trigonometry in Higher Dimensions and \\ a Diagonal Rule for 2-planes in Four-dimensional \\ space
}

\section{ABSTRACT}

We extend rational trigonometry to higher dimensions by introducing rational invariants between $k$-subspaces of $n$ dimensional space to give an alternative to the canonical or principal angles studied by Jordan and many others, and their angular variants. We study in particular the cross, spread and det-cross of 2-subspaces of four-dimensional space, and show that Pythagoras theorem, or the Diagonal Rule, has a natural generalization for such 2-subspaces.

Key words: rational trigonometry, subspaces, canonical angles, Diagonal rule, spread, cross

MSC2010: 51N25, 14Qxx, 14N20

\section{Introduction}

The notion of "angles" between two subspaces of a Euclidean space has seen many results going back to C. Jordan's foundational work in 1875 [7]. In the last fifty years, this idea has also seen considerable interest from statisticians and numerical analysts, who refer to "canonical or principal angles", as in [1], [2] and [3]. Applications of these "canonical angles" can be found in, for example, [4], [5], [8], [13] and [24].

Let us consider a general dot product space, by which we mean a vector space together with a non-degenerate symmetric bilinear form, or dot product, $u \cdot v$, although traditionally the topic involves only the classical case with the Euclidean inner product. We will refer to a subspace of dimension $k$ as a $k$-subspace.
Racionalna trigonometrija u višim dimenzijama i dijagonalno pravilo za 2-ravnine u 4-dimenzionalnom prostoru

\section{SAŽETAK}

Proširujemo racionalnu trigonometriju na više dimenzije tako da uvodimo racionalne invarijante između $k$ podprostora $n$-dimenzionalnog prostora. Dajemo alternativu kanonskim ili glavnim kutovima, koje su proučavali Jordan i mnogi drugi, te njihove varijante. Posebno proučavamo križni produkt, raspon i det-križni produkt 2podprostora 4-dimenzionalnog prostora i pokazujemo da Pitagorin teorem, ili dijagonalno pravilo, ima prirodnu generalizaciju za takve 2-podprostore.

Ključne riječi: racionalna trigonometrija, podprostori, kanonski kutovi, dijagonalno pravilo, raspon, križni produkt

Then for example in Jordan's theory, two 2-subspaces of four dimensional space determine in general two "angles" $\theta_{1}$ and $\theta_{2}$. The first is the smallest "angle" between nonzero vectors in the two spaces, while the second is the smallest "angle" between the orthogonal compliments in each subspace to the vectors creating the first "angle". For example if $P$ is the span of $(1,0,0,0)$ and $(0,1,0,0)$, while $R$ is the span of $(1,0,0, a)$ and $(0,1,0, b)$ for numbers $|a|<|b|$, then the usual description amounts to "angles" $\theta_{1}$ and $\theta_{2}$ specified (somewhat loosely) by

$\cos \theta_{1}=\frac{1}{\sqrt{1+a^{2}}} \quad$ and $\quad \cos \theta_{2}=\frac{\sqrt{1+a^{2}}}{\sqrt{1+a^{2}+b^{2}}}$

The work of Risteski and Trenčevski [14] suggests that if we multiply the cosines of the "canonical angles", then we 
may obtain the cosine of some new "geometrical angle" $\theta$ between the two given subspaces. In the special case where $\left(u_{1}, u_{2}, \cdots, u_{k}\right)$ and $\left(v_{1}, v_{2}, \cdots, v_{k}\right)$ are orthonormal bases of $k$-dimensional subspaces $P$ and $R$ of $R^{n}$, their formula reduces to

$\cos ^{2} \theta=\operatorname{det}\left(M^{T} M\right)$

in terms of the $k \times k$ matrix $M=\left[u_{i} \cdot v_{j}\right]$, although their original more general formulation had an error which was corrected by Gunawan, Neswan and Setya-Budhi in [6].

The reason that many of these concepts are put in quotes here is that this author is skeptical about the current "theory of real numbers" and most claims involving computations that require an "infinite amount" of computing time or power. But we do acknowledge that finite truncations of such procedures can have a very useful applied function. From our point of view even the usual definition of the "angle" between vectors $u$ and $v$ given by

$\cos \theta=\frac{u \cdot v}{|u||v|}$

is problematic in the realm of pure mathematics, on account of the "infinite amounts" of work needed to evaluate the square roots implicit in $|u|$ and $|v|$, and then to evaluate an inverse circular function.

We are interested in investigating a framework for pure mathematics that does not require us to pretend to obtain outputs from unending algorithms or computer programs.

Now many readers may very well not share this orientation, but we do hope that they can see that it might nevertheless be a legitimate logical position, and might actually steer us in natural and fruitful directions. This in fact leads us to rational trigonometry [15], [16], [17], [18]; to chromogeometry and associated triangle geometry [9], [10] and [19]; and to universal hyperbolic geometry, as in [20], [21], [22] and [23], so there is plenty of evidence that the approach has some merit.

To avoid "angles" therefore, we are going to re-frame the separation between two $k$-dimensional subspaces in an $n$ dimensional dot product space using an extension of a fundamental formula of rational trigonometry. Notice that we restrict to this more specific situation here, with subspaces of equal dimension. We will investigate some basic properties of rational invariants obtained from the characteristic polynomial of a cross matrix determined by two such subspaces, and in particular will prove a Pythagorean theorem, or Diagonal Rule result, for the special but quite interesting case of 2-subspaces in the four-dimensional Euclidean vector space $V^{4}$ of row vectors. The alternate new name for this historically important result reflects the fact that the Old Babylonian culture had a clear understanding of the geometry of a right triangle, more than 1000 years before Pythagoras was born, as discussed for example in [11].

\subsection{The basic vector orientation of rational trigonometry}

We begin by reviewing the basics of rational trigonometry, which was introduced in the planar situation in the author's book [15], and to more general situations in [16] and [19]. The main initial idea is to replace "transcendental metrical quantities" in Euclidean geometry with purely algebraic quantities. Thus the "distance" $|A, B|$ between two points $A$ and $B$ is replaced by the quadrance $Q(A, B)$, which is the sum of squares of the differences between the coordinates, and the "angle" $\theta(l, m)$ between two lines $l$ and $m$ is replaced by the spread $s(l, m)$, which may be thought of as the ratio of the opposite quadrance over the hypotenuse quadrance for any right triangle formed by the two lines, or the cross, which is the ratio of the adjacent quadrance over the hypotenuse quadrance.

More generally and explicitly, if a vector space over a field is given a symmetric bilinear form, or dot product, $u \cdot v$, then we define the quadrance of a vector $v$ to be the number

$Q(v) \equiv v \cdot v$.

A vector $v$ is null precisely when $Q(v)=0$. The cross between non-null vectors $u$ and $v$ is defined to be the number

$c(u, v) \equiv \frac{(u \cdot v)^{2}}{Q(u) Q(v)}$.

The spread between $u$ and $v$ is

$s(u, v) \equiv 1-c(u, v)=1-\frac{(u \cdot v)^{2}}{Q(u) Q(v)}$.

Since both the cross and spread are invariant under rescaling of either or both of the vectors, these quantities extend to metrical invariants between one-dimensional subspaces, just by considering spanning or direction vectors. So given one-dimensional subspaces $l$ and $m$ with respective spanning vectors $u$ and $v$, we may define the respective cross and spread between them as $c(l, m) \equiv c(u, v)$ and $s(l, m) \equiv s(u, v)$. These quantities are numbers in the given field.

For the vector space of row vectors of a given dimension $n$, the Euclidean symmetric bilinear form may be written in linear algebraic terms as

$u \cdot v=u v^{T}$.

A more general symmetric bilinear form is given by

$u \cdot v=u M v^{T}$ 
for some symmetric $n \times n$ matrix $M$, which is usually also assumed to be non-degenerate, meaning that $\operatorname{det} M \neq 0$.

Let us make an important additional observation: that since $u \cdot v=v \cdot u$, the (Euclidean) cross may be rewritten in this linear algebraic notation as

$c(u, v)=\frac{\left(u v^{T}\right)^{2}}{\left(u u^{T}\right)\left(v v^{T}\right)}=u v^{T}\left(v v^{T}\right)^{-1} v u^{T}\left(u u^{T}\right)^{-1}$

while the more general cross may be written as

$$
\begin{aligned}
c(u, v) & =\frac{\left(u M v^{T}\right)^{2}}{\left(u M u^{T}\right)\left(v M v^{T}\right)} \\
& =u M v^{T}\left(v M v^{T}\right)^{-1} v M u^{T}\left(u M u^{T}\right)^{-1} .
\end{aligned}
$$

How do these rational trigonometric definitions relate to the more familiar notions of distance and angle? One may attempt to "introduce a square-root" of the quadrance $Q(v)$ and so define a length $|v|$, but this is problematic on at least two counts; if working algebraically it generally requires an extension field, but analytically, this is a computational process that does not generally terminate, and so the ostensible outputs cannot be said to be well-defined (hence the quotes). Furthermore for other bilinear forms, as in Einstein's special theory of relativity, the quadrance can be negative, in which case we need also to introduce complex extension fields.

One may attempt to introduce an "inverse sine of a square root" of the spread, or the "inverse cosine of a square root" of the cross, to get an "angle", but again the transcendental aspect of these operations and the functions involved means that in practice only an approximation to an "ideal angle" is obtained after terminating the program to get an output in a finite amount of time. And in other geometries, as in Einstein's relativistic geometry, the quantities that appear as crosses and spreads need not be in the familiar interval $[-1,1]$, so "applying inverse circular functions" is not directly appropriate.

When we move to rational trigonometry as a framework for metrical geometry, many new possibilities for precision, clarity and generality open up. The quantities and their relations become algebraic and rational; we are not forced to assume a "real number framework" involving an algebraic structure that is rarely, if ever, set out logically and correctly in its entirety. We may aspire to obtain complete and correct results to metrical questions without the need to invoke symbolic arithmetic involving un-evaluated symbols such as $\pi, \sqrt{2}$, and $\cos 5$, or resorting to approximate values such as $3.1415,1.4142$ or 0.2837 etc.

\subsection{The affine laws of rational trigonometry}

To enunciate the main laws of rational trigonometry, a first step is to extend the above notions from the vector space framework to the corresponding affine framework. We may consider the affine space $A^{n}$, whose points are $n$-tuples $A=\left[a_{1}, a_{2}, \cdots, a_{n}\right]$ with entries from some field, but now with a translational symmetry as well as the linear transformational symmetry. To a pair of such affine points $A, B$ we may associate the displacement vector $v=\overrightarrow{A B}=\left(b_{1}-a_{1}, b_{2}-a_{2}, \cdots, b_{n}-a_{n}\right)$ in the usual fashion by taking differences of coordinates.

Suppose now that the associated vector space $V^{n}$ of row vectors $v=\left(x_{1}, x_{2}, \cdots, x_{n}\right)$ is given a non-degenerate symmetric bilinear form $u \cdot v$ as above. The quadrance between the affine points $A_{1}$ and $A_{2}$ is then correspondingly defined to be

$Q\left(A_{1}, A_{2}\right) \equiv \overrightarrow{A_{1} A_{2}} \cdot \overrightarrow{A_{1} A_{2}}$.

The affine line $A_{1} A_{2}$ determined by two points $A_{1}$ and $A_{2}$ is a null line precisely when $Q\left(A_{1}, A_{2}\right)=0$, that is when a direction vector for it is null.

The cross between non-null lines $l \equiv A_{1} A_{2}$ and $m \equiv B_{1} B_{2}$ is the number

$c(l, m) \equiv \frac{\left(\overrightarrow{A_{1} A_{2}} \cdot \overrightarrow{B_{1} B_{2}}\right)^{2}}{Q\left(A_{1}, A_{2}\right) Q\left(B_{1}, B_{2}\right)}$

while the spread between $l$ and $m$ is

$s(l, m) \equiv 1-c(l, m)=1-\frac{\left(\overrightarrow{A_{1} A_{2}} \cdot \overrightarrow{B_{1} B_{2}}\right)^{2}}{Q\left(A_{1}, A_{2}\right) Q\left(B_{1}, B_{2}\right)}$.

These are just the cross and spread of the associated direction vectors, and are independent of the choice of points lying on the two lines. If one or both of the lines involved are null, then the cross and spread are undefined, and statements involving them will be considered empty. Note also that we do not require the two lines to be meeting in order for these quantities to be defined.

Two non-null lines are perpendicular precisely when the cross between them is 0 , or equivalently when the spread between them is 1 . These conditions are just a restatement of the orthogonality of corresponding direction vectors with respect to the underlying bilinear form.

Here then are the five main laws of rational trigonometry for this metrical affine situation, where we use the convention that a triangle $\overline{A_{1} A_{2} A_{3}}$ is a set $\left\{A_{1}, A_{2}, A_{3}\right\}$ of three distinct points with quadrances $Q_{1} \equiv Q\left(A_{2}, A_{3}\right)$, $Q_{2} \equiv Q\left(A_{1}, A_{3}\right)$ and $Q_{3} \equiv Q\left(A_{1}, A_{2}\right)$, and spreads $s_{1} \equiv s\left(A_{1} A_{2}, A_{1} A_{3}\right), \quad s_{2} \equiv s\left(A_{2} A_{1}, A_{2} A_{3}\right)$ and $s_{3} \equiv$ $s\left(A_{3} A_{1}, A_{3} A_{2}\right)$. 


\section{Theorem 1 (Diagonal Rule or Pythagoras' theorem)}

The lines $A_{1} A_{3}$ and $A_{2} A_{3}$ are perpendicular precisely when

$Q_{1}+Q_{2}=Q_{3}$

Theorem 2 (Triple quad formula) The points $A_{1}, A_{2}$ and $A_{3}$ are collinear precisely when

$\left(Q_{1}+Q_{2}+Q_{3}\right)^{2}=2\left(Q_{1}^{2}+Q_{2}^{2}+Q_{3}^{2}\right)$

\section{Theorem 3 (Spread law)}

$\frac{s_{1}}{Q_{1}}=\frac{s_{2}}{Q_{2}}=\frac{s_{3}}{Q_{3}}$.

\section{Theorem 4 (Cross law)}

$\left(Q_{1}+Q_{2}-Q_{3}\right)^{2}=4 Q_{1} Q_{2}\left(1-s_{3}\right)$.

Note that the Cross law includes as special cases both the Triple quad formula and the Diagonal Rule when $s_{3}=0$ and $s_{3}=1$ respectively. The next result is the algebraic analog to the sum of the angles in a triangle formula.

\section{Theorem 5 (Triple spread formula)}

$\left(s_{1}+s_{2}+s_{3}\right)^{2}=2\left(s_{1}^{2}+s_{2}^{2}+s_{3}^{2}\right)+4 s_{1} s_{2} s_{3}$.

This last relation may be restated in terms of the corresponding crosses $c_{1} \equiv c\left(A_{1} A_{2}, A_{1} A_{3}\right), c_{2} \equiv c\left(A_{2} A_{1}, A_{2} A_{3}\right)$ and $c_{3} \equiv c\left(A_{3} A_{1}, A_{3} A_{2}\right)$ as follows.

\section{Theorem 6 (Triple cross formula)}

$\left(c_{1}+c_{2}+c_{3}\right)^{2}=4 c_{1} c_{2} c_{3}$.

With these laws we need no longer be restricted to Euclidean geometry metrically, but can apply our computations for example to relativistic geometries, since the main laws of rational trigonometry are valid independent of the particular bilinear form chosen (provided it is nondegenerate). And the computations work over a general field, notably the rational numbers with finite field extensions introduced as needed. Rational trigonometry extends also to finite fields, and indeed current work of Michael Reynolds is developing powerful software for such investigations.

Another very important advantage is that the above notions of quadrance and spread, introduced over a vector space over a field, have projective analogs when we consider the projective space associated to such a space. In this way we may assign notions of projective quadrance and projective spread to hyperbolic geometry in the Cayley Klein sense, yielding a more algebraic and general form of Universal hyperbolic geometry in which many new phenomenon are visible, and which has closer connections to relativistic physics on account of the fact that we can work uniformly inside or outside the light cone, as described in [20]. The essential projective nature of this approach means that elliptic geometry is captured by the same laws as for hyperbolic geometry - not just analogs where circular transcendental functions are replaced by hyperbolic ones as in the classical situation.

So we have enlarged geometry many-fold by moving to rational trigonometry for our metrical computations: we can work over the rational numbers; or over a finite field; we can consider arbitrary bilinear forms, including relativistic geometries; and we can create projective analogs of both elliptic and hyperbolic geometries simultaneously in the same general arena.

But another vista still beckons by more explicitly adopting a linear algebraic point of view. How might we extend rational trigonometry to higher dimensions, to consider metrical relations in the spirit of Jordan's "canonical or principal angles", between higher dimensional objects in such spaces? For example how does a symmetric bilinear form allow us to define rational metrical relations between $k$ dimensional subspaces of an $n$-dimensional vector $V^{n}$ or an associated affine space? And what theorems can we hope to find in these larger settings?

\section{Cross matrices, cross and det-cross}

Suppose that we have two $k \times n$ matrices $P$ and $R$, both of rank $k$, representing $k$-subspaces of the vector space $V^{n}$ over some field (with the rational numbers being the default choice), in the sense that the rows of each matrix are a basis of the corresponding $k$-subspace. Our aim is to introduce metrical invariants of the corresponding $k$-subspaces. The matrices $P P^{T}$ and $R R^{T}$ are both also of rank $k$, and so invertible $k \times k$ matrices. Then motivated by (2) and (3), we define the cross matrix of $P$ and $R$ to be

$C=C(P, R) \equiv P R^{T}\left(R R^{T}\right)^{-1} R P^{T}\left(P P^{T}\right)^{-1}$.

This is also a $k \times k$ matrix.

Now define the cross between $P$ and $R$ to be the normalized trace

$c=c(P, R) \equiv \frac{1}{k} \operatorname{tr} C$

and define the spread between $P$ and $R$ to be

$s=s(P, R) \equiv 1-c$.

Define the det-cross between $P$ and $R$ to be the number

$d(P, R) \equiv \operatorname{det}(C(P, R))$. 
The det-cross agrees with the square of the "cosine of the geometrical angle" of Risteski and Trenčevski as in (1), which we can see by considering the special case when $P$ and $R$ contain orthonormal vectors, so that $P P^{T}=R R^{T}=I$. But our introduction of the cross matrix yields a richer invariant that potentially gives us all the coefficients of the characteristic polynomial as invariants. These are some kinds of analogs of the canonical angles of Jordan.

The cross, spread and det-cross are numbers in the underlying field that depend on the matrices $P$ and $R$. We now demonstrate that they really depend only on the subspaces determined by $P$ and $R$. We first show that if we rearrange the rows of $P$ by an invertible linear transformation, then the new cross matrix is similar to the original, where two matrices $X$ and $Y$ are similar precisely when there is an invertible matrix $M$ with

$X=M Y M^{-1}$.

Theorem 7 If $P, R$ are both $k \times n$ matrices of rank $k$, and $M$ is an invertible $k \times k$ matrix, then $C(M P, R)=$ $M C(P, R) M^{-1}$ so that $c(M P, R)=c(P, R)$ and $d(M P, R)=$ $d(P, R)$.

Proof. If $M$ is an invertible $k \times k$ matrix then

$$
\begin{aligned}
C(M P, R) & =(M P) R^{T}\left(R R^{T}\right)^{-1} R(M P)^{T}\left((M P)(M P)^{T}\right)^{-1} \\
& =M P R^{T}\left(R R^{T}\right)^{-1} R P^{T} M^{T}\left(M P P^{T} M^{T}\right)^{-1} \\
& =M P R^{T}\left(R R^{T}\right)^{-1} R P^{T} M^{T}\left(M^{T}\right)^{-1}\left(P P^{T}\right)^{-1} M^{-1} \\
& =M P R^{T}\left(R R^{T}\right)^{-1} R P^{T}\left(P P^{T}\right)^{-1} M^{-1} \\
& =M C(P, R) M^{-1} .
\end{aligned}
$$

But since the trace and determinant of similar matrices are equal, we deduce that $c(M P, R)=c(P, R)$ and $d(M P, R)=$ $d(P, R)$.

Since the definition of the cross matrix is not quite symmetric in $P$ and $R$, a separate calculation is required to establish the effect of changing the basis for the row space of $R$.

Theorem 8 If $P, R$ are both $k \times n$ matrices of rank $k$, and $M$ is an invertible $k \times k$ matrix, then $C(P, M R)=C(P, R)$ so that $c(M P, R)=c(P, R)$ and $d(M P, R)=d(P, R)$.

Proof. If $M$ is an invertible $k \times k$ matrix then

$$
\begin{aligned}
C(P, M R) & =P(M R)^{T}\left((M R)(M R)^{T}\right)^{-1}(M R) P^{T}\left(P P^{T}\right)^{-1} \\
& =P R^{T} M^{T}\left(M R R^{T} M^{T}\right)^{-1} M R P^{T}\left(P P^{T}\right)^{-1} \\
& =P R^{T}\left(R R^{T}\right)^{-1} R P^{T}\left(P P^{T}\right)^{-1} \\
& =C(P, R) .
\end{aligned}
$$

So clearly $c(P, M R)=c(P, R)$ and $d(P, M R)=d(P, R)$.

There is one more important invariance with respect to these quantities: that they are unchanged under isometries of the Euclidean space with symmetric bilinear form $u \cdot v=u v^{T}$. Such an isometry is given by an $n \times n$ orthogonal matrix $Q$, with the property that $Q Q^{T}=I$, acting on the right on row vectors and so also on $k \times n$ matrices.

Theorem 9 If $P, R$ are both $k \times n$ matrices of rank $k$, and $Q$ is an orthogonal $n \times n$ matrix, then $C(P Q, R Q)=C(P, R)$ so that $c(M P, R)=c(P, R)$ and $d(M P, R)=d(P, R)$.

Proof. If $Q$ is an orthogonal $n \times n$ matrix then

$$
\begin{aligned}
& C(P Q, R Q)= \\
& =(P Q)(R Q)^{T}\left((R Q)(R Q)^{T}\right)^{-1}(R Q)(P Q)^{T}\left((P Q)(P Q)^{T}\right)^{-1} \\
& =P Q Q^{T} R^{T}\left(R Q Q^{T} R^{T}\right)^{-1} R Q Q^{T} P^{T}\left(P Q Q^{T} P^{T}\right)^{-1} \\
& =P R^{T}\left(R R^{T}\right)^{-1} R P^{T}\left(P P^{T}\right)^{-1} \\
& =C(P, R) .
\end{aligned}
$$

As before it follows that $c(P Q, R Q)=c(P, R)$ and $d(P Q, R Q)=d(P, R)$.

\section{The case of 2-subspaces of $V^{4}$}

Let us illustrate the above general notions in the specific case of 2-subspaces in $V^{4}$, the linear space of row vectors $v=\left(\begin{array}{llll}x_{1} & x_{2} & x_{3} & x_{4}\end{array}\right)$ whose elements belong to some field, which is typically the rational numbers. A twodimensional subspace, or 2-subspace, is the span of two linearly independent vectors, and may be specified by the $2 \times 4$ matrix with these vectors as the rows. So for example

$P=\left(\begin{array}{cccc}2 & -1 & 5 & 2 \\ 1 & 1 & 1 & 7\end{array}\right)$

represents the 2-subspace spanned by the two row vectors $\left(\begin{array}{llll}2 & -1 & 5 & 2\end{array}\right)$ and $\left(\begin{array}{llll}1 & 1 & 1 & 7\end{array}\right)$.

Clearly if we perform invertible elementary row operations on $P$, which is equivalent to multiplying on the left by an invertible matrix $M$, then we get another representative for the same subspace, for example

$$
P^{\prime}=\left(\begin{array}{cccc}
1 & 0 & 2 & 3 \\
0 & 1 & -1 & 4
\end{array}\right)=\left(\begin{array}{cc}
\frac{1}{3} & \frac{1}{3} \\
-\frac{1}{3} & \frac{2}{3}
\end{array}\right)\left(\begin{array}{cccc}
2 & -1 & 5 & 2 \\
1 & 1 & 1 & 7
\end{array}\right)
$$

which is the span of $\left(\begin{array}{llll}1 & 0 & 2 & 3\end{array}\right)$ and $\left(\begin{array}{llll}0 & 1 & -1 & 4\end{array}\right)$. We may write this relationship as $P^{\prime}=M P \sim P$ and observe that the two matrices represent the same subspace.

This generalizes the one-dimensional property of a onedimensional subspace being unchanged if a representative vector is multiplied by a non-zero scalar (or $1 \times 1$ matrix). To simplify notation we will often associate both the subspace and a defining matrix for it by the same letter. 
Example 1 Generalizing the first example in the Introduction, suppose that $P=\left(\begin{array}{llll}1 & 0 & 0 & 0 \\ 0 & 1 & 0 & 0\end{array}\right)$ and $R=$ $\left(\begin{array}{cccc}1 & 0 & x & y \\ 0 & 1 & z & w\end{array}\right)$. Then a computation shows that

$$
\begin{aligned}
C= & (P, R)=P R^{T}\left(R R^{T}\right)^{-1} R P^{T}\left(P P^{T}\right)^{-1} \\
= & \left(\begin{array}{ll}
(x w-y z)^{2}+x^{2}+y^{2}+z^{2}+w^{2}+1
\end{array}\right)^{-1} \\
& \left(\begin{array}{cc}
w^{2}+z^{2}+1 & -w y-x z \\
-w y-x z & x^{2}+y^{2}+1
\end{array}\right)
\end{aligned}
$$

so that

$c(P, R)=\frac{1}{2} \operatorname{tr}(C)=\frac{1}{2} \frac{x^{2}+y^{2}+z^{2}+w^{2}+2}{(x w-y z)^{2}+x^{2}+y^{2}+z^{2}+w^{2}+1}$

$s(P, R)=1-c(P, R)=\frac{1}{2} \frac{x^{2}+y^{2}+z^{2}+w^{2}+2(x w-y z)^{2}}{(x w-y z)^{2}+x^{2}+y^{2}+z^{2}+w^{2}+1}$

and

$d(P, R)=\operatorname{det} C=\frac{1}{(x w-y z)^{2}+x^{2}+y^{2}+z^{2}+w^{2}+1}$.

\section{Perpendicularity for 2-subspaces over the rational numbers}

In the vector case, two vectors $u$ and $v$ are perpendicular precisely when $s(u, v)=1$, or equivalently in terms of the cross $c(u, v)=0$. We now show that the same holds true for 2-subspaces in $V^{4}$, assuming we are working over the rational numbers. Note that the general situation, over a different field, may be different! The computations in the proof of this theorem are useful independently.

Theorem 10 (Perpendicular 2-subspace) Suppose that the underlying field is the rational numbers. If $P$ is a 2-subspace of $V^{4}$, then the only 2-subspace $T$ for which $c(P, T)=0$ is the orthogonal subspace $T=P^{\perp}$.

Proof. Since the cross is unchanged if we perform an orthogonal transformation or perform row reduction on representative vectors, the general case can be reduced to the case when $P=\left(\begin{array}{cccc}1 & 0 & 0 & 0 \\ 0 & 1 & 0 & 0\end{array}\right)$. Then by row reduction, any other 2-subspace $T$ has a representative matrix of one of the following kinds:

$$
\begin{array}{rlrl}
T_{12} & =\left(\begin{array}{llll}
1 & 0 & x & y \\
0 & 1 & z & w
\end{array}\right), & T_{13} & =\left(\begin{array}{llll}
1 & 0 & 0 & y \\
0 & 0 & 1 & w
\end{array}\right), \\
T_{14} & =\left(\begin{array}{llll}
1 & 0 & 0 & 0 \\
0 & 0 & 0 & 1
\end{array}\right), & T_{23} & =\left(\begin{array}{llll}
0 & 1 & 0 & y \\
0 & 0 & 1 & w
\end{array}\right), \\
T_{24}=\left(\begin{array}{llll}
0 & 1 & 0 & 0 \\
0 & 0 & 0 & 1
\end{array}\right), & T_{34}=\left(\begin{array}{llll}
0 & 0 & 1 & 0 \\
0 & 0 & 0 & 1
\end{array}\right) .
\end{array}
$$

We then compute that the various possibilities for the crosses with $P$ are:

$$
\begin{aligned}
& c\left(P, T_{12}\right)=\frac{1}{2} \frac{x^{2}+y^{2}+z^{2}+w^{2}+2}{(x w-y z)^{2}+x^{2}+y^{2}+z^{2}+w^{2}+1} \\
& c\left(P, T_{13}\right)=\frac{1}{2} \frac{w^{2}+1}{w^{2}+y^{2}+1} \\
& c\left(P, T_{14}\right)=\frac{1}{2} \\
& c\left(P, T_{23}\right)=\frac{1}{2} \frac{w^{2}+1}{w^{2}+y^{2}+1} \\
& c\left(P, T_{24}\right)=\frac{1}{2} \\
& c\left(P, T_{34}\right)=0 .
\end{aligned}
$$

Over the rational numbers, because squares are always positive, the only case when we can have $c(P, T)=0$ is the case of

$T=T_{34}=\left(\begin{array}{llll}0 & 0 & 1 & 0 \\ 0 & 0 & 0 & 1\end{array}\right)$.

This is the orthogonal subspace of $P$.

\section{A Diagonal Rule for 2-subspaces}

The Diagonal Rule, or Pythagoras' theorem, for vectors may be stated as follows.

Theorem 11 (One-dimensional Diagonal Rule) If $P$ and $R$ are perpendicular 1-subspaces of a two-dimensional space, that is for which $c(P, R)=0$, then for any 1subspace $T$ we have

$c(P, T)+c(R, T)=1$.

The reason why this somewhat unusual vector form for Pythagoras' theorem is equivalent to the usual affine one for triangles is as follows. Since the spread, or cross between two affine lines depends only on the direction vectors of those lines, they are unchanged if one or both of the lines are translated. So if we have a right triangle consisting of two 1-dimensional subspaces meeting perpendicularly at the origin, and another affine subspace lying in the same plane, the affine subspace may be translated to the origin where it becomes a 1-dimensional subspace, without any change to the respective spreads or crosses. Now we show that the one-dimensional result has a twodimensional analog. 
Theorem 12 (Two-dimensional Diagonal Rule) If $P$ and $R$ are perpendicular 2-subspaces of a four-dimensional space, that is for which $c(P, R)=0$, then for any 2subspace $T$ we have

$c(P, T)+c(R, T)=1$.

Proof. Without loss of generality, we can perform an orthogonal change of coordinates so that the first subspace $P$ is represented by the matrix

$P=\left(\begin{array}{llll}1 & 0 & 0 & 0 \\ 0 & 1 & 0 & 0\end{array}\right)$

The perpendicular subspace is then

$R=\left(\begin{array}{llll}0 & 0 & 1 & 0 \\ 0 & 0 & 0 & 1\end{array}\right)$

and we know that $c(P, R)=0$. Over the rational numbers we know that $R$ is the only 2 -subspace with the property that $c(P, R)=0$, but we do not require that here. Now any 2-dimensional subspace $T$, after row reduction, can be represented by one of the general matrices $T_{12}, T_{13}, T_{14}, T_{23}, T_{24}$ or $T_{34}$ displayed in the proof of the Perpendicular 2-subspace theorem above. That proof already established the crosses between $P$ and each of these matrices. We now similarly calculate the crosses between these matrices and the matrix $R$ :

$c\left(R, T_{12}\right)=\frac{1}{2} \frac{2 w^{2} x^{2}+w^{2}-4 w x y z+x^{2}+2 y^{2} z^{2}+y^{2}+z^{2}}{w^{2} x^{2}+w^{2}-2 w x y z+x^{2}+y^{2} z^{2}+y^{2}+z^{2}+1}$

$c\left(R, T_{13}\right)=\frac{1}{2} \frac{w^{2}+2 y^{2}+1}{w^{2}+y^{2}+1}$

$c\left(R, T_{14}\right)=\frac{1}{2}$

$c\left(R, T_{23}\right)=\frac{1}{2} \frac{w^{2}+2 y^{2}+1}{w^{2}+y^{2}+1}$

$c\left(R, T_{24}\right)=\frac{1}{2}$

$c\left(R, T_{34}\right)=1$.

Then using the preceding computations, we get

$$
\begin{aligned}
& c\left(P, T_{12}\right)+c\left(R, T_{12}\right)= \\
& \quad \frac{1}{2} \frac{w^{2}+x^{2}+y^{2}+z^{2}+2}{w^{2} x^{2}+w^{2}-2 w x y z+x^{2}+y^{2} z^{2}+y^{2}+z^{2}+1} \\
& \quad+\frac{1}{2} \frac{2 w^{2} x^{2}+w^{2}-4 w x y z+x^{2}+2 y^{2} z^{2}+y^{2}+z^{2}}{w^{2} x^{2}+w^{2}-2 w x y z+x^{2}+y^{2} z^{2}+y^{2}+z^{2}+1}=1
\end{aligned}
$$

$c\left(P, T_{13}\right)+c\left(R, T_{13}\right)=\frac{1}{2} \frac{w^{2}+1}{w^{2}+y^{2}+1}+\frac{1}{2} \frac{w^{2}+2 y^{2}+1}{w^{2}+y^{2}+1}=1$

$c\left(P, T_{14}\right)+c\left(R, T_{14}\right)=\frac{1}{2}+\frac{1}{2}=1$

$c\left(P, T_{13}\right)+c\left(R, T_{13}\right)=\frac{1}{2} \frac{w^{2}+1}{w^{2}+y^{2}+1}+\frac{1}{2} \frac{w^{2}+2 y^{2}+1}{w^{2}+y^{2}+1}=1$

$c\left(P, T_{13}\right)+c\left(R, T_{13}\right)=\frac{1}{2}+\frac{1}{2}=1$

$c\left(P, T_{13}\right)+c\left(R, T_{13}\right)=0+1=1$

In all cases the sum of the two crosses is

$c(P, T)+c(R, T)=1$.

\section{Two-dimensional subspaces of $V^{3}$}

Theorem 13 The spread between two 2-subspaces of $V^{3}$ is one half of the spread between the direction vectors of the subspaces perpendicular to their common meet.

Proof. Any two distinct 2-subspaces in $V^{3}$ meet in a onedimensional subspace. Let us assume, by performing an orthogonal transformation, that this common 1-subspace is the span of the vector $(1,0,0)$. By row reduction, the two 2-subspaces may be taken to be

$P=\left(\begin{array}{lll}1 & 0 & 0 \\ 0 & a & b\end{array}\right)$ and $R=\left(\begin{array}{lll}1 & 0 & 0 \\ 0 & c & d\end{array}\right)$.

Then

$$
\begin{aligned}
C & =C(P, R) \equiv P R^{T}\left(R R^{T}\right)^{-1} R P^{T}\left(P P^{T}\right)^{-1} \\
& =\left(\begin{array}{cc}
1 & 0 \\
0 & \frac{(a c+b d)^{2}}{\left(a^{2}+b^{2}\right)\left(c^{2}+d^{2}\right)}
\end{array}\right)
\end{aligned}
$$

with cross

$c=c(P, R)=\frac{1}{2} \operatorname{tr} C(P, R)=\frac{1}{2}\left(1+\frac{(a c+b d)^{2}}{\left(a^{2}+b^{2}\right)\left(c^{2}+d^{2}\right)}\right)$

and spread

$$
\begin{aligned}
s & =s(P, R)=1-c(P, R) \\
& =\frac{1}{2} \frac{(a d-b c)^{2}}{\left(a^{2}+b^{2}\right)\left(c^{2}+d^{2}\right)} .
\end{aligned}
$$

(Spread formula in $V^{3}$ )

This is one-half of the spread between the vectors $u=$ $(0, a, b)$ and $v=(0, c, d)$.

As an immediate corollary we obtain the following:

Proposition 1 For any 2-subspaces $P$ and $R$ of $V^{3}$ over the rational numbers the cross $c(P, R)$ and the spread $s(P, R)$ satisfy the inequalities

$\frac{1}{2} \leq c(P, R) \leq 1, \quad 0 \leq s(P, R) \leq \frac{1}{2}$. 
Proof. Over the rational number field, squares are always positive. So the inequality for $c(P, R)$ follows immediately from the formula for it in the previous proof, while the inequality for $s(P, R)$ follows from that.

\section{Acknowledgements}

The author would like to thank Hendra Gunawan for helpful discussions, and Michael Reynolds for his help in computing numerous examples of crosses and spreads in higher dimensional spaces, as well as the referee for useful suggestions.

\section{References}

[1] T.W. Anderson, An Introduction to Multivariate Statistical Analysis, John Wiley \& Sons, Inc., New York, 1958.

[2] A. BJÖRCK, G.H. Golub, Numerical methods for computing angles between linear subspaces, Math. Comp. 27 (1973), 579-594.

[3] C. DAVIES, W. KAHAN, The rotation of eigenvectors by a perturbation. III, SIAM J. Numer. Anal. 7 (1970), $1-46$.

[4] Z. DRMAČ, On principal angles between subspaces of Euclidean space, SIAM J. Matrix Anal. Appl. (electronic) 22 (2000), 173-194.

[5] A. Galántai, Cs.J. Hegedũs, Jordan's principal angles in complex vector spaces, Numer. Linear Algebra Appl. 13 (2006), 589-598.

[6] H. Gunawan, O. Neswan, W. Setya-Budhi, A formula for angles between two subspaces of inner product spaces, Beitr. Algebra Geom., Contributions to Algebra and Geometry, 46(2) (2005), 311-320.

[7] C. JORDAN, Essai sur la géométrie à $n$ dimensions, Bull. Soc. Math. France. 3 (1875), 103-174.

[8] A.V. Knyazev, M.E. Argentati, Principal angles between subspaces in an A-based scalar product: algorithms and perturbation estimates, SIAM J. Sci. Comput. 23 (2002), 2008-2040.

[9] N. Le, N.J. Wildberger, Incenter Circles, Chromogeometry, and the Omega Triangle, KoG 18 (2014), 5-18.

[10] N. Le, N.J. Wildberger, Universal Affine Triangle Geometry and Four-fold Incenter Symmetry, KoG 16 (2012), 63-80.

[11] D. Mansfield, N.J. Wildberger, Plimpton 322 is Babylonian Exact Sexagesimal Trigonometry, Historia Math. 44(4) (2017), 395-419.
[12] J. Miao, A. Ben-Israel, Product cosines of angles between subspaces, Linear Algebra Appl. 237/238 (1996), 71-81.

[13] V. RAKočEvić, H.K. Wimmer, A variational characterization of canonical angles between subspaces, J. Geometry 78 (2003), 122-124.

[14] I.B. Risteski, K.G. Trenčevski, Principal values and principal subspaces of two subspaces of vector spaces with inner product, Beitr. Algebra Geom., Contributions to Algebra and Geometry 42(1) (2001), 289-300.

[15] N.J. Wildberger, Divine Proportions: Rational Trigonometry to Universal Geometry, Wild Egg Books, Sydney, 2005, http: //wildegg.com

[16] N.J. WildBerger, Affine and projective metrical geometry, 2007, http://arxiv.org/abs/math/ 0701338

[17] N.J. Wildberger, A Rational Approach to Trigonometry, Math Horiz. 15(2)(2007), 16-20.

[18] N.J. WildBerger, One dimensional metrical geometry, Geom. Dedicata 128(1) (2007), 145-166.

[19] N.J. Wildberger, Chromogeometry and relativistic conics, KoG 13 (2009), 43-50.

[20] N.J. Wildberger, Universal Hyperbolic Geometry I: Trigonometry, Geom. Dedicata 163(1) (2013), 215-274.

[21] N.J. WILDBERGER, Universal Hyperbolic Geometry II: A pictorial overview, KoG 14 (2010), 3-24.

[22] N.J. WILDBERGER, Universal Hyperbolic Geometry III: First steps in projective triangle geometry, $\mathrm{KoG}$ 15, (2011), 25-49.

[23] N.J. Wildberger, A. Alkhaldi, Universal Hyperbolic Geometry IV: Sydpoints and Twin Circumcircles, KoG 16 (2012), 43-62.

[24] H.K. Wimmer, Canonical angles of unitary spaces and perturbations of direct complements, Linear Algebra Appl. 287 (1999), 373-379.

\section{N J Wildberger}

orcid.org/0000-0003-3503-6495

e-mail: n.wildberger@unsw.edu.au

School of Mathematics and Statistics UNSW

Sydney 2052 Australia 